\title{
Expression of angiogenesis-related genes in right atrial appendages from patients with advanced myocardial ischemia - preliminary results
}

Józefa Dąbek¹, Joanna Głogowska-Ligus², Andrzej Bochenek ${ }^{3}$

1Department of Cardiology, Medical University of Silesia, Katowice, Poland 2Department of Epidemiology, Medical University of Silesia, Katowice, Poland $31^{\text {st }}$ Cardiosurgery Chair and Clinic, Medical University of Silesia, Katowice, Poland

Submitted: 28 June 2011

Accepted: 3 October 2011

Arch Med Sci 2013; 9, 3: 540-543

DOI: 10.5114 /aoms.2013.35419

Copyright (C 2013 Termedia \& Banach

\section{Abstract}

Introduction: The most frequent cause of myocardial ischemia is atherosclerotic lesions which narrow coronary vessels leading to impaired blood flow or their total occlusion.

Material and methods: Using HG-U133A oligonucleotide microarrays (Affymetrix) we studied the expression levels of angiogenesis-related genes in patients with different types of heart failure. We analyzed the RNA from right atrial appendages from patients: 1) with advanced coronary disease and myocardial infarction history, 2) with advanced stable coronary disease and no infarction history, and 3) after surgery due to mitral stenosis, but with no coronary vessel lesions. Results: Analysis of the data from oligonucleotide microarrays allowed identification of 2 genes (ENG and NPPB) differentiating the examination grups. Conclusions: Analysis of the expression profile of genes involved in angiogenesis, carried out using data obtained from examined individuals' samples, suggests that necrosis accompanying myocardial infarction is a significant factor leading to elevated expression levels of genes involved in neoangiogenesis.

Key words: myocardial ischemia, angiogenesis, microarray analysis.

\section{Introduction}

The most frequent cause of myocardial ischemia is atherosclerotic lesions which narrow coronary vessels leading to impaired blood flow or their total occlusion. The latter is caused by destabilization of atherosclerotic plaque and thrombus formation and results in myocardial infarction [1]. The infarct with its accompanying necrosis is a pathological condition favoring angiogenesis, consisting in the formation of thin-walled vascular structures lined with endothelial cells.

This HG-U133A oligonucleotide microarray-based study was aimed at assessing the expression pattern of angiogenesis-related genes in right atrial appendages from the following patients: 1) with advanced coronary disease and myocardial infarction history, 2) with advanced stable coronary disease and no infarction history, and 3) after surgery due to mitral stenosis, but with no coronary vessel lesions.

\author{
Corresponding author: \\ Assist. Prof. Józefa Dąbek \\ Department of Cardiology \\ Medical University of Silesia \\ 45 Ziołowa St \\ 40-635 Katowice, Poland \\ Phone: + 48323598530 \\ E-mail: jdabek@sum.edu.pl
}




\section{Material and methods}

The study was designed according to good clinical practice (GCP) rules consistent with the Declaration of Helsinki. The project was positively evaluated (decision NN-6501-223/I/04) by the Bioethics Commission of the Medical University of Silesia in Katowice.

The material from right atrial appendages from three patients who underwent planned surgery at the $1^{\text {st }}$ Cardiosurgery Clinic, Medical University of Silesia, was analyzed. The following patients were qualified to participate in the study: one with advanced stable angina and history of inferior wall myocardial infarction (sample 2), one with advanced stable angina and no history of myocardial infarction (sample 7), and one after surgery due to mitral stenosis and without coronary lesions (sample 11). A regular sinus rhythm in the examined patients was concluded both during physical examination and when interpreting the ECG. During cardiac surgery in extracorporeal circulation a part of the right atrium was removed, stored immediately in liquid nitrogen and subsequently used in the study.

RNA extraction from intrasurgical tissue sample homogenates was carried out using TRIzol (Invitrogen). RNA obtained was analyzed using HG-U133A (Affymetrix) oligonucleotide microarrays. The results were analyzed with the help of RMA Express software.

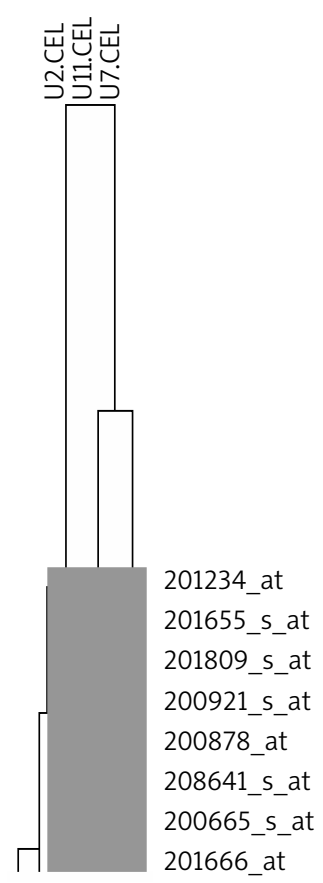

Figure 1. Hierarchical clustering of 229 angiogenesisrelated transcripts analyzed

Using literature data and the accessed Affymetrix database, from among 22283 transcripts present on the HG-U133A microarray we were able to isolate 229 angiogenesis-related genes. Subse-

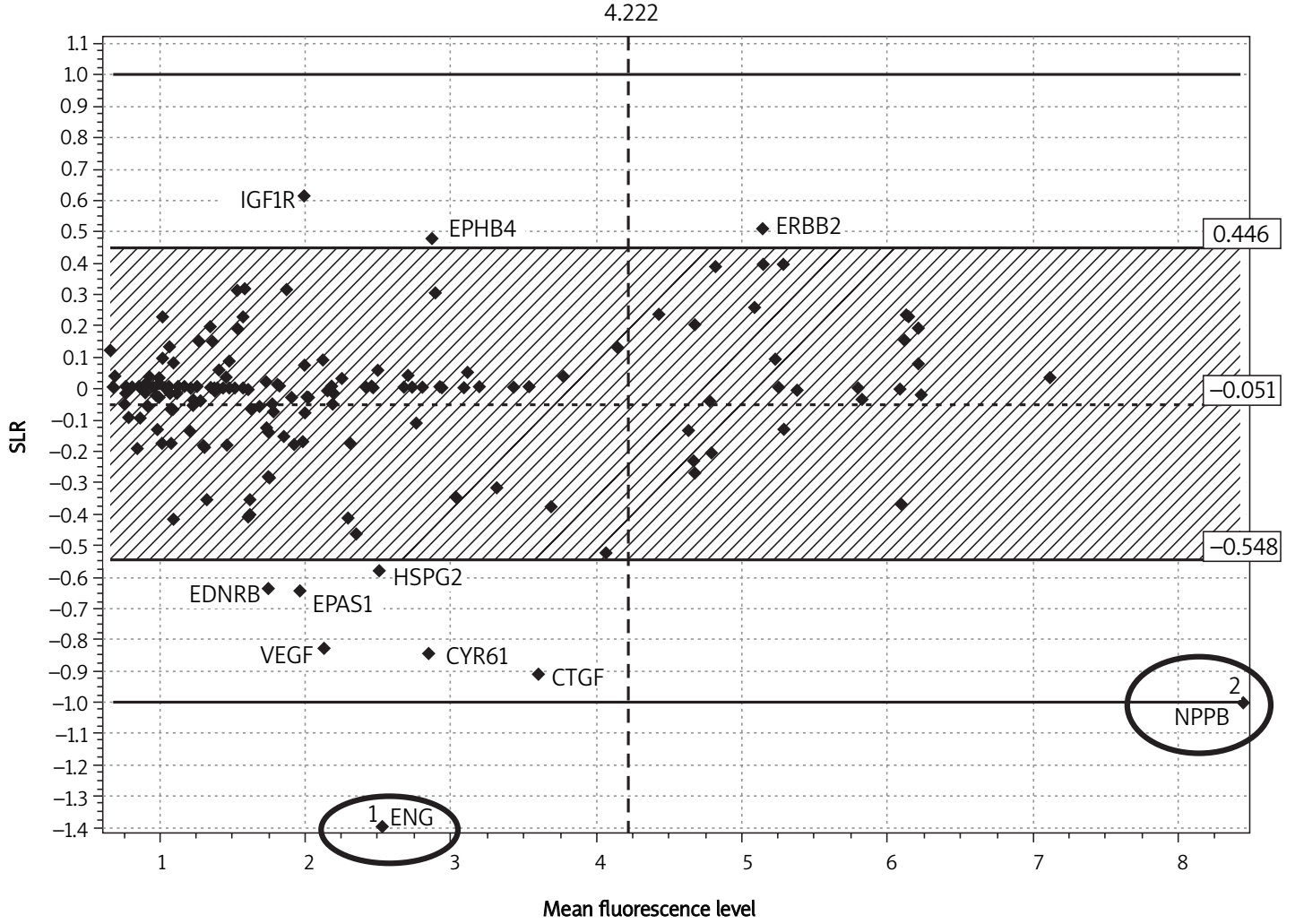

Figure 2. Typing of genes that differentiate between a patient with advanced angina and history of myocardial infarction (U2) from a patient with stable advanced angina and no history of myocardial infarction (U7) and from a patient who underwent surgery due to mitral stenosis, but without coronary lesions (U11) 
quently they were analyzed using a hierarchical clustering method (Cluster v 3.0). In applying a modified Bland-Altman method, the following criteria were adopted: 1) differentiation of signal log ratio (SLR) of particular transcripts, 2) minimum value of fluorescence level assuring usefulness of a designated differential transcript in QRT-PCR analysis, and 3) the so-called "biological criterion" meaning the desired minimum value of the SLR coefficient of the differential transcript $\left(S L R_{\min }= \pm 1\right)$.

\section{Results}

To assess similarity of transcriptomes of the examined samples hierarchical clustering analysis was performed. It demonstrated that the expression profile of angiogenesis-related genes in sample 2 is different from the profiles of the remaining samples (Figure 1).

Using the Bland-Altman method the following genes were found that The analysis demonstrated two genes differentiating the examined groups (Figure 2). They were endoglin (ENG) and proBNP precursor (NPPB). Their expression levels were more than two-fold higher in sample 2 than in samples 7 and 11 .

\section{Discussion}

Angiogenesis is a complex process of forming novel blood vessels, regulated by a number of factors, either activating or inhibiting this process. A survey of literature data confirms that heartbeat disturbances, and more specifically atrial fibrillation, may affect changes in gene expression $[2,3]$. The patients referred to in the report did not present, neither during interview nor during hospitalization in the Cardiology Clinic, any signs of heartbeat disturbances, including atrial fibrillation. As to the impact of other cardiovascular disease risk factors (tobacco consumption, hyperlipidemia and concomitant diseases), we shall be able to formulate an answer after conducting and carefully analyzing QRT-PCR results from a large group of patients.

The results of our study demonstrate significantly elevated expression of NPPB and ENG genes in the patient with a history of myocardial infarction, as compared to the two remaining subjects examined.

NPPB is an inhibitor of the renin-angiotensinaldosterone system (RAAS) responsible for preserving volumetric and pressure homeostasis of circulating blood. NPPB release occurs in response to an increase in blood pressure, whence its significant elevation in subjects with myocardial damage present [4]. Data published so far concerning expression of the circulating molecular precursor of proBNP in patients with a history of myocardial infarction and in cardiogenic shock show its elevated levels [5]. Similar results were obtained in our study.

Endoglin promotes angiogenesis by reinforcing activation of transforming growth factor $\beta 1$ (TGF $\beta 1$ ), which is preponderantly expressed on proliferating endothelial cells. Elevated expression of endoglin observed in connection with myocardial infarction is linked with activation of ENG/ALK-1 signaling pathways activated in endothelial cells during hypoxia, which occurs both in vivo and in vitro. These results prove that the ENG/ALK-1 pathway is one of the factors regulating the activity of myocardial cells during their post-infarction adaptation [6]. The results obtained in the present study, which show elevated expression of the ENG gene in a patient with myocardial infarction history, seem to corroborate the quoted observation.

Our analysis also revealed a series of candidate genes for the purpose of differentiating between similar examined samples. They are genes with elevated expression in subjects with a history of myocardial infarction (EPAS1, VEGF, CTGF, EDNRB, HSPG2 and CYR61) as well as genes with downregulated expression in such subjects (EPHB4, ERBB2 and IGF1R). It needs to be stressed that these are preliminary results and that it is necessary to conduct further quantitative studies (QRT-PCR) corroborating the results presented herein.

In conclusion, analysis of the expression profile of genes involved in angiogenesis, carried out using data obtained from examined individuals' samples, suggests that necrosis accompanying myocardial infarction is a significant factor leading to elevated expression levels of genes involved in neoangiogenesis.

\section{Acknowledgments}

Molecular work was performed in the Department of Molecular Biology, School of Pharmacy and Division of Laboratory Medicine, Medical University of Silesia in Katowice.

The study was conducted after obtaining written consent from the local Bioethics Commission (NN6501-223/1/04, amended May 22, 2007). The experimental part of this study was performed at the laboratories of the Department of Molecular Biology, the Silesian Medical University in Katowice.

\footnotetext{
References

1. Isordia-Salas I, Mendoza-Valdez AL, Almeida-Gutiérrez E, Borrayo-Sánchez G. Genetic factors of the hemostatic system in young patients with myocardial infarction. Cir Cir 2010; 78: 93-7.

2. Ohki R, Yamamoto K, Ueno S, et al. Gene expression profiling of human atrial myocardium with atrial fibrilla-
} 
tion by DNA microarray analysis. Int J Cardiol 2005; 102:

233-8.

3. Lamirault G, Gaborit N, Le Meur N, et al. Gene expression profile associated with chronic atrial fibrillation and underlying valvular heart disease in man. J Mol Cell Cardiol 2006; 40: 173-84.

4. Das BB. Plasma B-type natriuretic peptides in children with cardiovascular diseases. Pediatr Cardiol 2010; 31: 1135-45.

5. Hejmdal A, Boesgaard S, Lindholm MG, Goetze JP. B-type natriuretic peptide and its molecular precursor in myocardial infarction complicated by cardiogenic shock. J Card Fail 2007; 13: 184-8.

6. Tian F, Zhou AX, Smits AM, et al. Endothelial cells are activated during hypoxia via endoglin/ALK-1/SMAD1/5 signaling in vivo and in vitro. Biochem Biophys Res Commun 2010; 392: 283-8. 